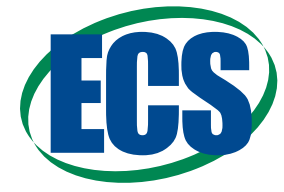

Jes Focus Issue on Lithium-Sulfur Batieries: Materials, Mechanisms, Modeling, and Applications

\title{
Polysulfides Formation in Different Electrolytes from the Perspective of X-ray Absorption Spectroscopy
}

\author{
Robert Dominko, ${ }^{\mathrm{a}, \mathrm{b}, \mathrm{z}}$ Alen Vizintin, (1) ${ }^{\mathrm{a}}$ Guiliana Aquilanti, ${ }^{\mathrm{c}}$ Lorenzo Stievano, (i) ${ }^{\mathrm{d}, \mathrm{e}}$ \\ Maria Joseph Helen, ${ }^{\mathrm{f}}$ Anji Reddy Munnangi, ${ }^{\mathrm{f}}$ Maximilian Fichtner, ${ }^{\mathrm{f}}$ and Iztok Arcon ${ }^{\mathrm{g}, \mathrm{h}}$ \\ ${ }^{a}$ Department of Materials Chemistry, National Institute of Chemistry, 1000 Ljubljana, Slovenia \\ ${ }^{b}$ Faculty of Chemistry and Chemical Technology University of Ljubljana, 1000 Ljubljana, Slovenia \\ ${ }^{c}$ Elettra-Sincrotrone Trieste S.C.p.A., 34149 Basovizza, Trieste, Italy \\ ${ }^{d}$ Institut Charles Gerhardt Montpellier, CNRS UMR 5253, Université de Montpellier, 34095 Montpellier, France \\ ${ }^{e}$ Réseau sur le Stockage Electrochimique de l'Energie, CNRS FR 3459, 80039 Amiens Cedex, France

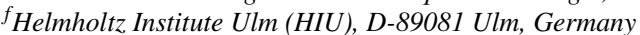 \\ ${ }^{g}$ University of Nova Gorica, 5000 Nova Gorica, Slovenia \\ ${ }^{h}$ Institut Jožef Stefan, 1000 Ljubljana, Slovenia
}

\begin{abstract}
Li-S batteries are promising energy storage technology for the future, however there two major problems remained which need to be solved before successful commercialization. Capacity fading due to polysulfide shuttle and corrosion of lithium metal are directly connected with the type and quantity of electrolyte used in the cells. Several recent works show dependence of the electrochemical behavior of Li-S batteries on type of the electrolyte. In this work we compare and discuss a discharge mechanism of sulfur conversion in three different electrolytes based on measurements with sulfur K-edge XAS. The sulfur conversion mechanism in the ether based electrolytes, the most studied type of solvents in the Li-S batteries, which are enabling high solubility of polysulfides are compared with the fluorinated ether based electrolytes with a reduced polysulfide solubility and in carbonate based electrolytes with the sulfur confined into a ultramicroporous carbon. In all three cases the sulfur reduction proceeds through polysulfide intermediate phases with a difference on the type polysulfides detected at different steps of discharge.

(C) The Author(s) 2017. Published by ECS. This is an open access article distributed under the terms of the Creative Commons Attribution 4.0 License (CC BY, http://creativecommons.org/licenses/by/4.0/), which permits unrestricted reuse of the work in any medium, provided the original work is properly cited. [DOI: 10.1149/2.0151801jes]

(cc) BY
\end{abstract}

Manuscript submitted September 19, 2017; revised manuscript received November 14, 2017. Published December 20, 2017. This paper is part of the JES Focus Issue on Lithium-Sulfur Batteries: Materials, Mechanisms, Modeling, and Applications.

Electrification of road transport has increased the pressure on materials' scientists to improve performance of the current Li-ion batteries and to develop new advanced high energy battery technologies. ${ }^{1}$ Among several post lithium-ion technologies, the lithium sulfur (Li-S) batteries are recognized as the most promising for commercialization in the near future. A combination of lithium and sulfur in the electrochemical cell corresponds to the theoretical energy density of 2600 $\mathrm{Wh} / \mathrm{kg}$, while the maximum practically accessible energy density is predicted to be close to $600 \mathrm{Wh} / \mathrm{kg}^{2,3}$ This is much higher compared to Li-ion batteries and besides that, sulfur is inexpensive and naturally abundant. Nevertheless, problems related to the solubility of polysulfides in the electrolyte and the related redox shuttle phenomena cause short cycle life, potential safety problems, poor cycling efficiency, and relative fast self-discharge. Additional problems of Li-S batteries are very low electronic conductivity of the both end members in the discharge and charge process (i.e. sulfur and $\mathrm{Li}_{2} \mathrm{~S}$ ) and extensive corrosion of the metal lithium anode.

Different directions how to improve Li-S battery cycle life have been explored, like synthesis of the optimized porous host matrices with active sites for polysulfide anchoring, ${ }^{4,5}$ design and optimization of separators which can effectively suppress polysulfide cross communication between electrodes ${ }^{6,7}$ and protection of lithium by artificial SEI ${ }^{8}$ or by additives. ${ }^{9}$ While most of the research was performed in the binary mixture of solvents using alkyl ethers (glymes) and heterocyclic acetyl (dioxolane), less attention has been paid to the development of new electrolytes for Li-S batteries. ${ }^{10}$ Reasons for that are nested in the requirements which should be fulfilled in the development of new formulations. First of all, electrolytes used in the electrochemical cells must have good ionic conductivity and electrochemical stability in a wide potential window. It is desired that they are non-toxic and environmentally friendly, non-reactive with active species, non-corrosive with cell housing and they should enable the sufficient dissolution of the supporting salt.

${ }^{\text {zE} E-m a i l: ~ R o b e r t . D o m i n k o @ k i . s i ~}$
Electrolytes used in Li-S batteries typically enable the dissolution of polysulfides which act as redox mediator species in the conversion reaction and significantly improve the kinetics of the electrochemical reaction. With that, solubility of sulfur and polysulfides has a direct influence on the discharge mechanism due to differences in dissolution and solvation of polysulfides. It is well accepted that the electrochemical mechanism of sulfur reduction in Li-S batteries is a stepwise process where a different equilibrium states between sulfur, polysulfides and $\mathrm{Li}_{2} \mathrm{~S}$ exists. ${ }^{11}$ In general, the discharge mechanism can be described by using two to three equilibrium states i.e. equilibrium between sulfur and long chain polysulfides dissolved into the electrolyte, equilibrium between long and short chain polysulfides and equilibrium between polysulfides and precipitated $\mathrm{Li}_{2} \mathrm{~S}$ (or even nonsoluble $\left.\mathrm{Li}_{2} \mathrm{~S}_{2}\right) .{ }^{12}$ The potential of the high voltage plateau, typically found in the starting part of the electrochemical curve, is controlled by the dissolution of sulfur into the electrolyte. Thus, one can expect that the equilibrium potential during high voltage plateau will vary depends on the type of electrolyte used in the electrochemical characterization. The low voltage plateau corresponds to the formation of non-soluble $\mathrm{Li}_{2} \mathrm{~S}$, which starts to precipitates when the solubility of $\mathrm{Li}_{2} \mathrm{~S}$ is exceeded. The understanding of the sulfur conversion mechanism is crucial to further improve electrochemical behavior of Li-S batteries.

Numerous analytical techniques have been applied for the characterization of Li-S batteries in order to better understand the mechanism of sulfur conversion within the Li-S battery. Different groups ${ }^{13-15}$ showed that operando XRD can be used as an excellent tool to characterize the formation and consumption of a crystalline active species for instance $\mathrm{Li}_{2} \mathrm{~S}$ and different allotropes of sulfur. Early studies were not concentrated on the detection of polysulfides, since they were considered as amorphous species detected in the background of the XRD spectra. Recently, J. Conder et al. ${ }^{16}$ showed the direct observation of lithium polysulfides adsorbed on the surface of a glass fiber separator in the Li-S battery during operation by means of operando X-ray diffraction (XRD). Although synchrotron XRD enables detection of polysulfides, ${ }^{16,17}$ much better understanding can be obtained by using 
sulfur K-edge X-ray absorption spectroscopy (XAS). With a difference to XRD, XAS is sensitive to coordination of the selected atom and can differentiate between different local coordination and oxidation states. This technique is currently used by several groups ${ }^{18-20}$ and it provides the most comprehensive picture of reaction mechanism(s) inside the battery since it can precisely differentiate sulfur species in the cathode during discharge and charge process. In most of the reported works, analysis of the near edge structure of the $\mathrm{X}$-ray absorption spectrum (XANES) is used for discussion of the mechanism and only a few papers discuss also extended X-ray absorption fine structure (EXAFS) part of the XAS spectra. The reason for that are sulfate groups in the salt (i.e. LiTFSI) or in the solvent (i.e. sulfonyl functional groups) composing the electrolyte. Nevertheless, different sulfur compounds (sulfur, polysulfides, sulfur radicals, $\mathrm{Li}_{2} \mathrm{~S}$, molecules with C-S bonds, sulfite, and sulfate) formed in the electrochemical cell can be identified by characteristic energy position of the sulfur edge and pre-edge resonances in the range between $2468 \mathrm{eV}$ for sulfur radicals ${ }^{21}$ to $2478 \mathrm{eV}$ for sulfate ${ }^{22}$ (corresponding to sulfur with the highest oxidation state). As a reference database for polysulfides, different stoichiometric mixtures between sulfur and lithium were synthesized and measured by XAS and ${ }^{7} \mathrm{Li}$ NMR. ${ }^{19}$ Measured spectra of sodium and lithium polysulfides represent a reference database that can be used for analysis of spectra obtained during battery operation. All intermediate states of polysulfides can be identified by the ratio between the pre-peak at $2470.2 \mathrm{eV}$ (characteristic pre-peak for polysulfides) and the sulfur peak at $2472 \mathrm{eV}$ typical of elemental sulfur, since the relative intensity of the pre-peak is proportional to the relative amount of $\mathrm{Li}$ in $\mathrm{Li}_{2} \mathrm{~S}_{\mathrm{y}}$. Experimental results ${ }^{19}$ show that obtained spectra can be in general described with a contribution from the long chain polysulfides $\left(\mathrm{Li}_{2} \mathrm{~S}_{\mathrm{y}}\right.$ with $\mathrm{y}$ $\geq 6$ ) where the intensity of pre-peak is relatively weak compared to sulfur peak and with mid- to short chain polysulfides $\left(\mathrm{Li}_{2} \mathrm{~S}_{\mathrm{y}}\right.$ with y $\leq 4)$, where the ratio between pre-peak and sulfur peak is smaller but still not equal to that expected for $\mathrm{Li}_{2} \mathrm{~S}_{2}$. Early theoretical work predicted slight variations in the position of the lithium polysulfide pre-edge peak in the adsorption spectra. ${ }^{23}$ By using Resonant Inelastic X-ray Scattering (RIXS) no shifts were observed for the set of reference samples, neither within operando measurement. ${ }^{24}$ Nevertheless, information gathered from operando XAS measurements can serve as a reliable indicator of changes within the Li-S battery upon operation.

Other types of spectroscopic analytical techniques are predominantly based on higher wavelengths such as Raman spectroscopy, ${ }^{25}$ infrared spectroscopy ${ }^{26}$ and UV-Vis spectroscopy. ${ }^{27,28}$ The latter has demonstrated to be a useful technique for qualitative and quantitative detection of polysulfides in the separator (detection of polysulfides which diffuse/migrate out from the cathode composite). In several of our works ${ }^{22,27,28}$ we highlighted the sensitivity of the UV-VIS analytical technique, showing that it offers hints about the polysulfide formation mechanism which occurs in the bulk of the cathode. However, this spectroscopic tool for the study of polysulfide mechanism is based on the prediction that polysulfides are soluble into the electrolyte. Besides that, typically a high ratio between electrolyte and sulfur is used in the experiment. This technique is less powerful in the current trends where electrolytes with a reduced solubility of polysulfides are predicted to be a key for successful commercialization of Li-S batteries. Similarly, reduced applicability of UV-Vis spectroscopy is also in the carbonate based electrolytes where sulfur has to be confined in narrow (nanometer size) pores, well passivated on the surface to protect direct reaction between carbonate solvents and polysulfides.

In this contribution we discuss Li-S battery mechanisms in different electrolyte systems by means of XAS. The cases of sulfur reduction in ether based electrolytes enabling high solubility of polysulfides, in fluorinated ether based electrolytes with a reduced polysulfide solubility and in carbonate based electrolytes with the sulfur confined into a ultramicroporous carbon to avoid the polysulfide solubility are discussed based on measurements with sulfur K-edge XAS.

\section{Experimental}

X-ray absorption spectra were recorded at the sulfur K-edge in fluorescence-detection mode at the XAFS beamline of synchrotron Elettra (Basovizza, Trieste). ${ }^{29}$ A Si (111) double crystal monochromator was used with a resolution of about $0.4 \mathrm{eV}$ at $2.5 \mathrm{keV}$. Higherorder harmonics were effectively eliminated by using a double flat silica mirror placed at a grazing angle of $8 \mathrm{mrad}$. The intensity of the monochromatic X-ray beam before the sample was measured by a $30 \mathrm{~cm}$-long ionization chamber detector, filled with a mixture of 30 mbar of $\mathrm{N}_{2}$ and 1970 mbar of He. The fluorescence signal was detected with a SDD detector. The spectra were measured within the interval of -150 to $730 \mathrm{eV}$ relative to the $\mathrm{S}$ K-edge $(2472 \mathrm{eV})$. In the XANES region, equidistant energy steps of $0.2 \mathrm{eV}$ were used, whereas for the higher energy region, equidistant $k$ steps of $0.03 \AA^{-1}$ were adopted, with an integration time of $5 \mathrm{~s}$ per point, resulting in the total time for one spectrum of $65 \mathrm{~min}$. The experimental setup of the beamline for the operando XAS experiments is described in details elsewhere. ${ }^{30}$ The first spectrum was measured on the asprepared battery, and a series of 27 following spectra were collected in operando mode during battery discharge at $\mathrm{C} / 30$ rate. The exact energy calibration was established with an absorption measurement on the native sulfur, where the maximum of the pre-edge peak was set to $2472.0 \mathrm{eV}^{19}$ In order to limit self-absorption effects in sulfur K-edge XANES spectra, the cathode was prepared in the initial state with a relatively low amount of sulfur. The setup of the measurement, cell and battery configuration were same as in our previous operando Li-S battery study, presented in more detail in the Supporting Information of the paper. ${ }^{12}$ The analysis of the XANES spectra was performed with the IFEFFIT program package ATHENA. ${ }^{31}$ No self-absorption correction was applied to the measured XAS spectra, because the composition of the cathode (ratio between sulfur, lithium, electrolyte, and $\mathrm{CB}$ ) could not be determined precisely enough.

The electrodes for the operando XAS measurements were prepared by mixing the carbon sulfur composite with conductive additive and binder as explained in detail in our previous publications. ${ }^{12,19}$ operando XANES measurements were performed in the two electrode set up with as prepared cathode and lithium metal (Aldrich) which were separated by glass fiber separator wetted with different types of electrolytes. Sulfur conversion in the mesoporous carbon was studied in the $1 \mathrm{M}$ LiTDI TEGDME:DOL ${ }^{12}$ electrolyte and in the 1 M LiTFSI TTFE:DOL (1,2-(1,1,2,2-tetrafluoroethoxy)ethane and 1,3-dioxolane $)^{32}$ electrolyte. Carbonate based electrolyte (1 $\mathrm{M} \mathrm{LiPF}_{6}$ in EC:DMC) were used in the combination with the ultramicroporous carbon. Galvanostatic cycling was performed in a modified Swagelok cell with thin beryllium window. ${ }^{12}$ Cell were discharged at a current density of $\mathrm{C} / 30\left(55.7 \mathrm{~mA} \mathrm{~g}^{-1}\right)$ to $1.5 \mathrm{~V}$ by using Biologic SP 200 galvanostat/potentiostat.

\section{Results and Discussion}

Sulfur reduction proceeds through disproportionation reactions of different polysulfide species which can be in the solid state form or dissolved in the electrolyte. Understanding their formation and conversion to $\mathrm{Li}_{2} \mathrm{~S}$ requires studies under real-time conditions since with that we can avoid the artefacts from post treatment and we can get insight to the real working mechanism(s). ${ }^{17} \mathrm{XAS}$ provides details on oxidation state (XANES part of the spectrum) and atomic distribution (EXAFS part of the spectrum). Contributions of different sulfur compounds in the cathode (sulfur, Li-polysulfides with different chain lengths, $\mathrm{Li}_{2} \mathrm{~S}$ ) in the XANES spectra can be identified by characteristic energy positions and shape of the sulfur absorption edge and pre-edge resonances. ${ }^{12}$ The energy position of the pre-edge resonance in elemental sulfur is at $2472 \mathrm{eV}$. Lithium polysulfides, containing a reduced form of sulfur with oxidation state $\mathrm{S}^{2-}$, can be identified by a characteristic pre-peak at $2470.2 \mathrm{eV}$ together with a sulfur peak at $2472 \mathrm{eV}$. The XANES spectrum of crystalline $\mathrm{Li}_{2} \mathrm{~S}$ exhibits the first maximum at sulfur absorption edge at $2473 \mathrm{eV}$. An additional spectral 


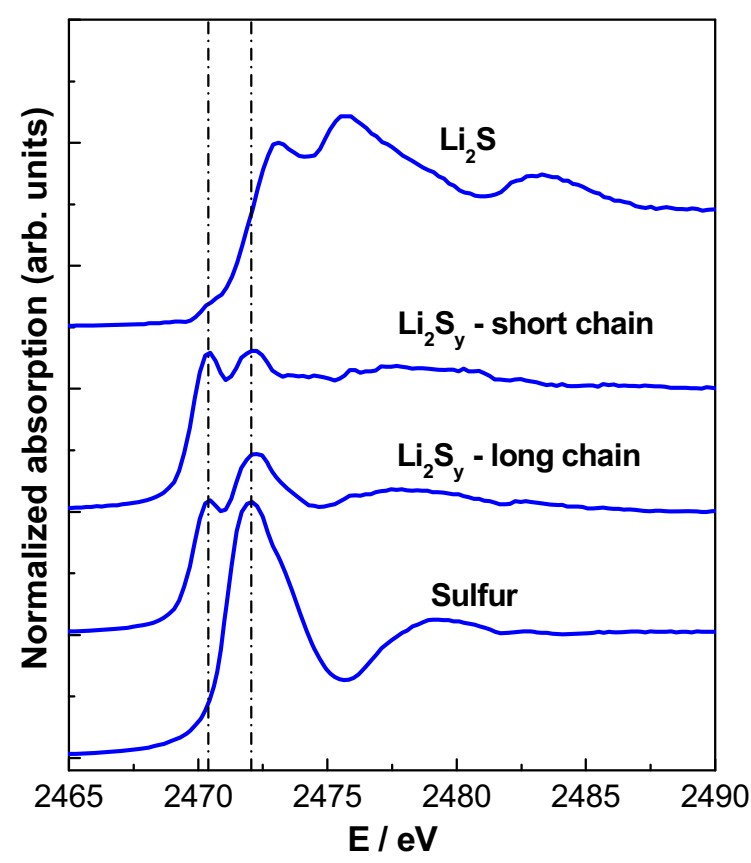

Figure 1. Sulfur K-edge XANES spectra of sulfur compounds present in the $\mathrm{Li}-\mathrm{S}$ battery: sulfur, $\mathrm{Li}_{2} \mathrm{~S}_{\mathrm{y}}$ polysulfides (long and short chains), and lithium sulfide $\mathrm{Li}_{2} \mathrm{~S}$. The two polysulfides spectra, $\left(\mathrm{Li}_{2} \mathrm{~S}_{\mathrm{y}}-\right.$ long chains, and $\mathrm{Li}_{2} \mathrm{~S}_{\mathrm{y}}$ - short chains) are representative spectra for a mixture of long chains polysulfides, and a mixture of short chains polysulfides, respectively. The vertical dotted lines at 2470.2 and $2472.0 \mathrm{eV}$ mark characteristic pre-peaks in the $\mathrm{Li}_{2} \mathrm{~S}_{y}$ and sulfur reference spectra.

signature was described in the literature ${ }^{21}$ with pre-edge resonances at $2468 \mathrm{eV}$, corresponding to $\mathrm{S}_{3}{ }^{\bullet-}$ radical. Using a combination of spectral components shown in Figure 1 one can describe mechanism of sulfur conversion in different electrolytes by using a linear combination analysis (LCA). Some other contributions can be identified like formation of radicals, ${ }^{21}$ formation of $\mathrm{H}_{2} \mathrm{~S}$ or $\mathrm{C}-\mathrm{S}$ bond due to nucleophilic reaction of polysulfides with solvent molecules.

Mechanism in the ether based electrolytes.-Alkyl ethers and heterocyclic acetyl represent a family of solvents stable towards polysulfides and with different polysulfide solubilities. This family of solvents is frequently applied in the battery research and it offers high conversion of sulfur into $\mathrm{Li}_{2} \mathrm{~S}$ due to soluble intermediate species. The solubility of polysulfides depends on the type of solvents as well as on the concentrations of salt(s) and additives. ${ }^{33-35}$ It is well accepted that polysulfides act as redox mediator species improving rate capability of Li-S batteries. Improved sulfur conversion is related to the solubility of sulfur in the heterocyclic acetal. ${ }^{34}$ However, high solubility of polysulfides leads to polysulfide shuttle phenomena due to diffusion/migration of polysulfides out from the cathode composite. Additionally, soluble polysulfides increase the viscosity of the electrolyte and reduce the lithium mobility in the electrolyte. More recently, fluorinated ether solvents showed improved Li-S battery behavior with more stable capacity retention. ${ }^{36-38}$ This is attributed to the reduced solubility of polysulfides which also enables Li-S battery cycling with reduced amount of electrolyte $\left(<5 \mu \mathrm{L} / \mathrm{mg}_{\mathrm{S}}\right)$ since the physicochemical properties of the electrolyte are stable during cycling.

At this step the open question is how different polysulfide solubility influences the reaction mechanism of sulfur conversion. We studied those mechanisms by different analytical techniques; however, in this work we will focus on the differences observed by using sulfur Kedge XAS. The mechanism of sulfur conversion in the mixture of alkyl ethers and heterocyclic acetyl (solvent mixture of TEGDME and DOL) was studied in the presence of $1 \mathrm{M}$ LiTDI salt and such a system enabled us to measure and analyze complete XAS spectra (XANES and EXAFS part). ${ }^{12}$ All sulfur K-edge XANES spectra measured in operando mode can be thoroughly described as a linear combination of three components (sulfur, polysulfides and $\mathrm{Li}_{2} \mathrm{~S}$ ). Two different rates of sulfur conversion into polysulfides were detected in the starting part of the electrochemical reduction, which can be correlated to the different voltage plateaus observed in the electrochemical curve (Figure 2a). At the beginning of the reduction process, during the high voltage plateau, we observed fast consumption of elemental sulfur and increase of polysulfide concentration. The conversion rate of sulfur changed once the potential started to decrease from high voltage plateau towards to the low voltage plateau. The maximum ratio of polysulfides within the cathode composite was detected at the beginning of the low voltage plateau, where precipitation of $\mathrm{Li}_{2} \mathrm{~S}$ starts. During the low voltage plateau, three phases coexist, with slow reduction of the remaining elemental sulfur and with linear increase of $\mathrm{Li}_{2} \mathrm{~S}$ quantity accompanied by the linear decrease of polysulfides quantity. At the end of the reduction process sulfur is completely consumed and we observed approximately $70 \%$ of $\mathrm{Li}_{2} \mathrm{~S}$ in co-existence with polysulfides. Interestingly, all operando spectra could be fitted with only one polysulfide
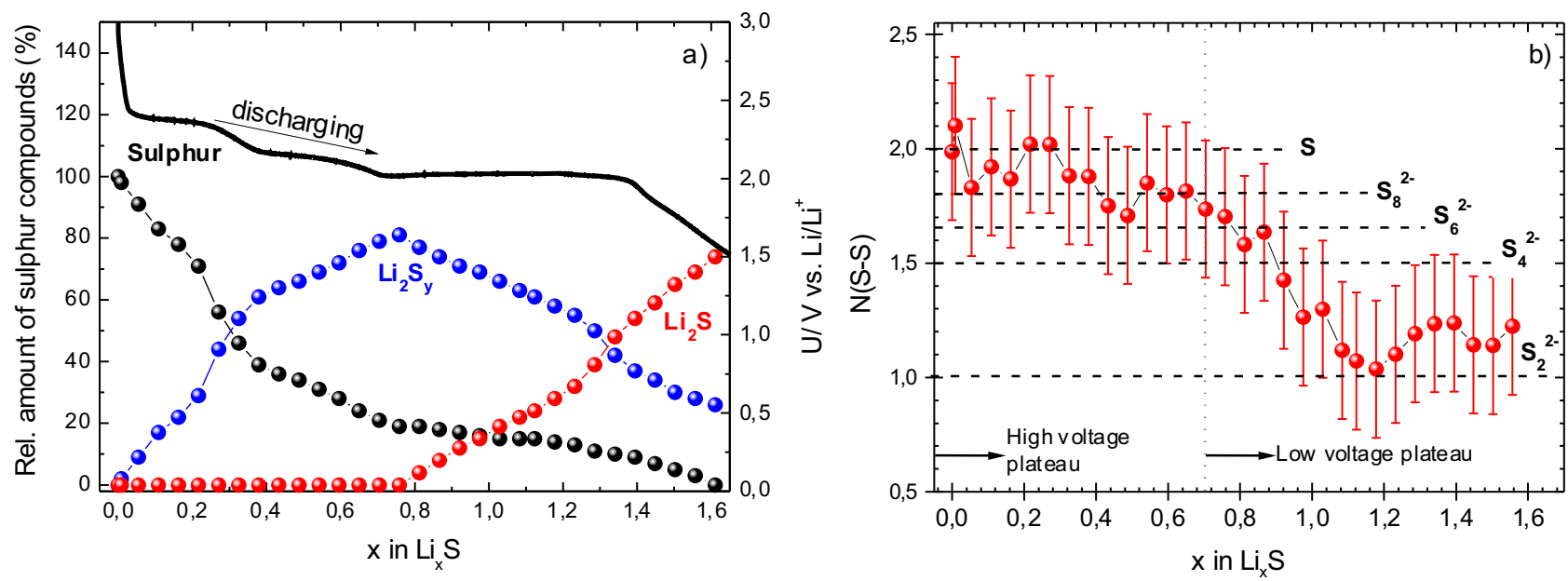

Figure 2. a) Electrochemical curve obtained during discharge in the $1 \mathrm{M}$ LiTDI TEGDME:DOL electrolyte and the relative amounts of three sulfur compounds (sulfur, polysulfides and $\mathrm{Li}_{2} \mathrm{~S}$ ) determined with a linear combination fit of XANES spectra; $b$ ) variation of the average $\mathrm{S}$ coordination number during the first discharge as determined by analysis of the EXAFS part of spectra measured in the 1 M LiTDI TEGDME:DOL electrolyte. Reprinted with permission from Ref. 12. Copyright (2015) American Chemical Society. 


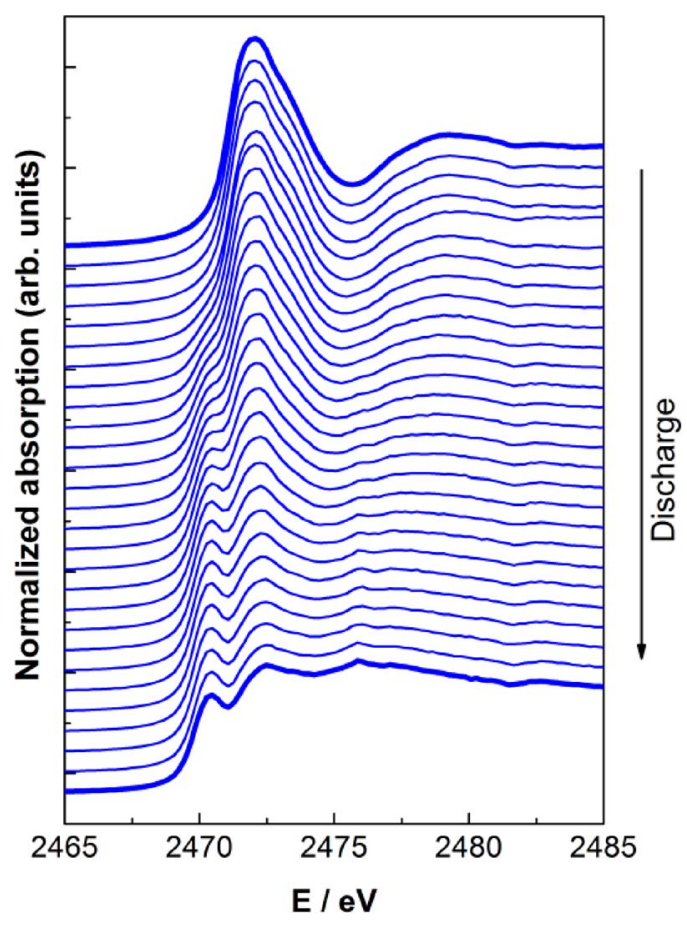

Figure 3. In operando sulfur K-edge XANES spectra measured on as prepared $\mathrm{Li}-\mathrm{S}$ battery with sulfur infiltrated ultramicroporous carbon within $1 \mathrm{M} \mathrm{LiPF}_{6} \mathrm{EC}: \mathrm{DEC}$ electrolyte at discharge $\mathrm{C} / 30$.

standard that suggests presence of an equilibrium state of polysulfides within the electrochemical cell.

A sulfur-free electrolyte enabled the possibility to study the transformation of the local environment of sulfur by quantitative EXAFS analysis. It was performed for the $\mathrm{S}-\mathrm{S}$ first coordination shell and the variation of the average coordination number of S-S component is shown in Figure $2 b$. A detailed explanations of the procedures used for EXAFS analysis are given in our previous work. ${ }^{12}$ The variation of the average coordination number of S-S component shows gradual decrease of coordination number during high voltage plateau. At the end of the high voltage plateau, the average coordination number is decreased from 2 to $1.6(2)$ which corresponds to the average coordination number typical of $\mathrm{S}_{6}{ }^{2-}$. At the beginning of the low voltage plateau, a steeper decrease of average coordination number is observed with a rapid decrease to values between $\mathrm{S}_{4}{ }^{2-}$ and $\mathrm{S}_{2}{ }^{2-}$. EXAFS analysis has confirmed the gradual shortening of polysulfide chain length as the average coordination number of the S-S component decreases during the discharge process.

Sulfur and polysulfide solubility in the fluorinated based ethers are several orders of magnitude lower compared to linear glymes. ${ }^{32}$ That can be explained by different potential of the high voltage plateau typically found at $2.2 \mathrm{~V}$ versus lithium reference and not at $2.4 \mathrm{~V}$ as observed in the electrolyte solutions with linear glymes. Nevertheless, a detailed analysis of Li-S battery discharge mechanism in $1 \mathrm{M}$ LiTFSI TTFE:DOL electrolyte by using $\mathrm{S}$ K-edge XANES shows co-existence of polysulfides with elemental sulfur through entire discharge process. ${ }^{32}$ Precipitation of $\mathrm{Li}_{2} \mathrm{~S}$ starts at the beginning of the low voltage plateau and this equilibrium is not influenced by choice of electrolyte. ${ }^{32}$ In the LCF analysis of the operando XANES spectra measured on batteries with ether electrolyte used, only a single reference polysulfide spectrum was sufficient to describe polysulfide contribution during the entire process of discharge. That is pointing out on the existence of equilibrium of different polysulfides during the reduction process. This equilibrium depends on the different parameters and for each working system, reference spectra for polysulfides needs to be extracted out from in the operando XANES spectra.

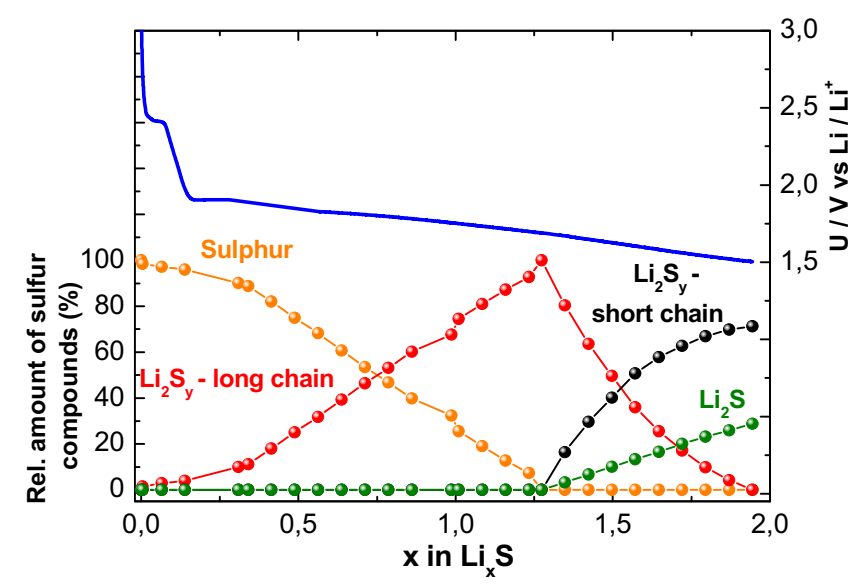

Figure 4. Electrochemical curve obtained during discharging at a current rate of $\mathrm{C} / 30$ and the relative amount of the four sulfur compounds (sulfur, long and short chains $\mathrm{Li}_{2} \mathrm{~S}_{\mathrm{y}}$, and $\mathrm{Li}_{2} \mathrm{~S}$ ) during discharge, determined with a linear combination fit of $\mathrm{S}$ K-edge XANES spectra measured at different operando states of the battery using four reference XANES profiles (sulfur, $\mathrm{Li}_{2} \mathrm{~S}_{y}$ with long and with short chains, and $\mathrm{Li}_{2} \mathrm{~S}$ ).

Mechanism in the carbonate solvent based electrolytes.-U1tramicroporous carbons with a pore diameter smaller than $0.7 \mathrm{~nm}$ gained considerable interest as host matrix for sulfur in Li-S batteries since such sulfur confinement shows excellent electrochemical stability even in the carbonate based solvents. ${ }^{39-41}$ It is well accepted that soluble polysulfides react with the carbonate based electrolytes via a nucleophilic addition or substitution reactions ${ }^{42}$ leading to extensive degradation of electrolyte. In this work ultramicroporous carbon derived from coconut shell containing predominately micropores with a diameter of $0.5 \mathrm{~nm}$ has been used as host matrix for sulfur. In a previous work some of us proved excellent electrochemical properties in the $1 \mathrm{M} \mathrm{LiPF}_{6} \mathrm{EC}: \mathrm{DMC}$ electrolyte. Cycling stability over 400 cycles was demonstrated with excellent Coulombic efficiency. That was possible due to the effective separation of sulfur confined in ultramicropores and due to formation of SEI on the surface of coconut shell derived carbon in the first cycle. A short plateau in the first cycle close to $2.4 \mathrm{~V}$ corresponds to the formation of SEI on the surface of carbon/sulfur composite which effectively separate electrolyte and carbon sulfur composite. Once a protective SEI is formed it enables lithium diffusion and prevents diffusion of polysulfides into the electrolyte; consequently no soluble polysulfides can be detected during cycling. The confined sulfur without a direct contact with the electrolyte shows a different reduction mechanism compared to that observed in ether based solvents, where the starting reduction potential depends on the sulfur solubility. In the previous work ${ }^{39}$ where a detailed analysis performed by ex situ XPS sputter profiling on electrodes removed from batteries stopped at different states of charge showed the direct conversion of sulfur to $\mathrm{Li}_{2} \mathrm{~S}_{2} / \mathrm{Li}_{2} \mathrm{~S}$. In this work we evaluate the reduction mechanism by operando sulfur K-edge XAS. operando XANES spectra measured during battery discharge are shown in Figure 3. Principle component analysis (PCA) ${ }^{31}$ of the whole set of spectra shows that a linear combination of four different components is required to completely describe each XANES spectrum in the series. Components with a physical meaning are sulfur, long and short chain Li-polysulfides $\mathrm{Li}_{2} \mathrm{~S}_{\mathrm{y}}$ and lithium sulfide $\mathrm{Li}_{2} \mathrm{~S}$ (Figure 4). The reference $\mathrm{Li}_{2} \mathrm{~S}$ XANES spectrum was obtained in our previous operando Li-S battery study, ${ }^{12}$ while the remaining three reference XANES profiles are extracted directly from the set of operando XANES spectra of this battery. The elemental sulfur XANES spectrum is obtained from the spectrum of the as-prepared battery, which contained only elemental sulfur. No difference was observed between bulk sulfur and sulfur infiltrated into the ultramicropores of carbon derived from coconut shells. The two Lipolysulfides spectra, $\left(\mathrm{Li}_{2} \mathrm{~S}_{\mathrm{y}}-\right.$ long chain, and $\mathrm{Li}_{2} \mathrm{~S}_{\mathrm{y}}-$ short chain $)$ are 

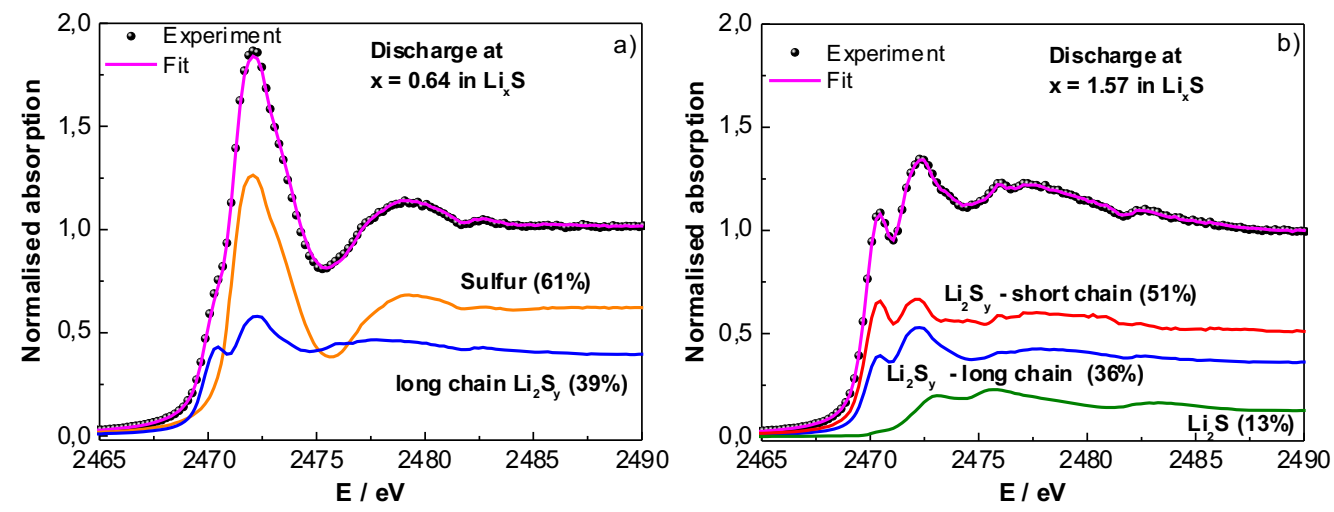

Figure 5. Sulfur K-edge XANES spectra of a Li-S battery measured in operando in two intermediate states during discharge: a) 556 min and b) 1371 min. Dots: experiment; dashed magenta line: best fit with linear combination four reference XANES profiles (sulfur, long chain polysulfides, short chain polysulfides, and $\left.\mathrm{Li}_{2} \mathrm{~S}\right)$, plotted below.

representative spectra for a mixture of long chain polysulfides $\left(\mathrm{S}_{8}{ }^{2-}\right.$, $\left.\mathrm{S}_{6}{ }^{2-}\right)$, and a mixture of short chain polysulfides $\left(\mathrm{S}_{4}{ }^{2-}, \mathrm{S}_{2}{ }^{2-}\right)$, respectively. All operando XANES spectra measured in other intermediate states of the battery during discharge can be completely described as linear combination of these four reference spectra. The quality of the linear combination fits (LCF) is demonstrated in Figure 5. In this way the evolution of the relative amounts of the sulfur species (sulfur, two different types of Li-polysulfides $\mathrm{Li}_{2} \mathrm{~S}_{\mathrm{y}}$, and lithium sulfide $\mathrm{Li}_{2} \mathrm{~S}$ ) in the cathode during discharge are determined with high precision, with an uncertainty below $1 \%$ for all components. In contrast to our previous work, ${ }^{12,19}$ two different polysulfide standards are needed in LCF analysis, as indicated by PCA analysis, to completely describe XANES spectra of all intermediate states of the battery. That points out a different mechanism of sulfur reduction in the ultramicropores.

End of discharge at $1.5 \mathrm{~V}$ contains $29 \%$ of $\mathrm{Li}_{2} \mathrm{~S}$, while the number of reacted $\mathrm{Li}$ suggests almost complete conversion of sulfur to $\mathrm{Li}_{2} \mathrm{~S}$. This difference is due to the irreversible consumption of $\mathrm{Li}$ for the formation of a protective SEI film on the surface of the ultramicroporous carbon. Efficient SEI formation is required to prevent formation of $\mathrm{H}_{2} \mathrm{~S}$ due to the presence of $\mathrm{HF}$ in the electrolytes with $\mathrm{LiPF}_{6}$ salt. Formation of SEI can be associated to charge consumed during plateau close to $2.4 \mathrm{~V}$, namely XANES spectra during this period of charge are almost unchanged. Once when voltage drops to $1.9 \mathrm{~V}$ vs. lithium reference a fast increase of long chain- $\mathrm{Li}_{2} \mathrm{~S}_{\mathrm{y}}$ was observed. A composition of $\mathrm{Li}_{1.27} \mathrm{~S}$ represents a verge, where contribution from long chain- $\mathrm{Li}_{2} \mathrm{~S}_{\mathrm{y}}$ starts to decrease accompanying with a formation of short chain- $\mathrm{Li}_{2} \mathrm{~S}_{\mathrm{y}}$ and $\mathrm{Li}_{2} \mathrm{~S}$. After this point, the relative amount of $\mathrm{Li}_{2} \mathrm{~S}$ is linearly increasing and it reaches $29 \%$ at the end of the discharge. Increase of short chain- $\mathrm{Li}_{2} \mathrm{~S}_{\mathrm{y}}$ polysulfides is not linear which points out potential shortening of polysulfides, however the end of discharge composition of the electrode is a mixture between $\mathrm{Li}_{2} \mathrm{~S}(29 \%)$ and $\mathrm{Li}_{2} \mathrm{~S}_{2}(71 \%)$ as it was already suggested by XPS. ${ }^{39}$ This final composition determined from the XAS measurements was expected also from the charge consumed during the reduction, where a monotonous voltage drop from 1.9 to $1.5 \mathrm{~V}$ mainly corresponds to the reduction of sulfur to $\mathrm{Li}_{2} \mathrm{~S}$ and $\mathrm{Li}_{2} \mathrm{~S}_{2}$ and only very minor part to additional passivation. A more complete conversion to $\mathrm{Li}_{2} \mathrm{~S}$ is probably hindered by space confinement within the pores with a dimension less than $0.7 \mathrm{~nm}$.

Mechanistic changes influenced by different electrolytes.-XAS measurements confirmed the presence of polysulfides in all three studied electrolytes with some differences. Sulfur reduction proceeds in all three electrolytes through polysulfide intermediates with a difference that sulfur almost completely disappears in the first part of discharge in the $1 \mathrm{M}$ LiTDI TEGDME:DOL electrolyte. On the contrary, in electrolytes characterized by a sparing solubility for polysulfides or in carbonate based electrolytes, only part of the sulfur is converted into polysulfides during high voltage plateau, the rest being continuously consumed by the end of discharge. Differences in the reaction mechanism in the first part of the reduction process are therefore strictly related to the solubility of sulfur and polysulfides in the electrolyte: with higher solubility, we observed faster sulfur conversion into polysulfides. Interestingly also higher solubility of sulfur and polysulfides determines the potential of the high voltage plateau, which is at 2.4 $\mathrm{V}$ in the TEGDME:DOL electrolyte and at $2.2 \mathrm{~V}$ in TTFE:DOL electrolyte, where solubility of sulfur and polysulfides is a few orders of magnitude lower. In carbonate based electrolytes due to the effective separation of sulfur confined in ultramicropores and due to protective nature of SEI, there is no solubility of sulfur and polysulfides in the electrolyte and the high voltage plateau is absent in the electrochemical reduction curve. Nevertheless, independent on whether the electrolyte composition enables the presence of soluble polysulfides or not, the Li-S battery discharge mechanism proceeds through polysulfides, which can be in the solid state form or dissolved. A conversion process of the polysulfides from long chain to short chain is observed in all tested electrolytes, even though in ether based electrolytes we were not able to clearly distinguish between different chain lengths. That was made possible by using ultramicroporous carbon, where the clear presence of long chain polysulfides at the beginning of discharge was observed, followed by the presence of short chain polysulfides (most likely $\mathrm{Li}_{2} \mathrm{~S}_{2}$ composition) in the second part of the discharge process. The equilibrium potential of $\mathrm{Li}_{2} \mathrm{~S}$ formation is at similar voltage in both ether based electrolytes used in this study while in the confined space, $\mathrm{Li}_{2} \mathrm{~S}$ formation starts at much lower voltage. That can be attributed to the limited space for volumetric changes that are required for formation of $\mathrm{Li}_{2} \mathrm{~S}$. The latter is also the most probable reason for relative low ratio of $\mathrm{Li}_{2} \mathrm{~S}$ formed at the end of discharge compared to end of discharge in the TEGDME:DOL electrolyte.

Slight difference in the sulfur conversion mechanism between three investigated electrolytes can be used for better understanding of advantages and drawbacks of each electrolyte. Mixture of alkyl ethers and heterocyclic acetyl (TEGDME and DOL) enables high solubility of sulfur and polysulfides. That influences physical properties of electrolyte (i.e. viscosity, lithium mobility ... ) and consequently higher amounts of electrolyte are required to achieve high capacity and long cycle life, but electrolyte quantity higher then $3 \mathrm{~mL}$ per $1 \mathrm{~g}$ of sulfur is not acceptable for commercial purposes.

Sulfur and polysulfide solubility in the sparingly soluble solvents (i.e. fluorinated ethers) is for several orders of magnitude lower compared to glymes, what allows decrease of electrolyte quantity and low solubility of polysulfides still has a function of redox mediators. The weakest point of using fluorinated ethers is increased resistance film on lithium surface, but due to the possibility of having working Li-S battery with very low amount of electrolyte this type of electrolyte is of particular interest for commercially available cells. 
Carbonate solvents are known as much better passivation agents for lithium compared to ethers and since sulfur infiltrated ultra microporous carbons can be used in the combination carbonate based electrolyte, this direction offers new unexplored field of Li-S batteries. Major difficulties are lower voltage due to difference in the mechanism and lower capacity due to volumetric constrains inside pores, while benefits of that should be taken from Li-ion battery technology.

\section{Conclusions}

In the ether based electrolytes the sulfur conversion and formation of polysulfides occurs predominantly during the high voltage plateau. The maximum ratio of polysulfides is detected at the beginning of the low voltage plateau, where the precipitation of $\mathrm{Li}_{2} \mathrm{~S}$ begins. The high voltage potential in the ether based electrolytes is determined by the solubility of sulfur and polysulfides. During the low voltage plateau a coexistence of three different oxidation states of sulfur is typically found, while the potential of low voltage plateau depends on the equilibrium between polysulfides and $\mathrm{Li}_{2} \mathrm{~S}$. End of discharge, sulfur is converted to polysulfides which are in the coexistence with $\mathrm{Li}_{2} \mathrm{~S}$ as a major product of sulfur conversion. The fluorinated based electrolytes enable much lower solubility of sulfur and polysulfides in the electrolyte, the mechanism of sulfur conversion is not affected by decreased solubility. Although solubility of polysulfides is several orders of magnitude lower, polysulfides equilibrium with elemental sulfur is dominating high voltage plateau and similar as in the ether based electrolytes, the precipitation of $\mathrm{Li}_{2} \mathrm{~S}$ starts with a low voltage plateau.

Reversible sulfur reduction into $\mathrm{Li}_{2} \mathrm{~S}$ in the carbonate based electrolytes is possible only with confinement of sulfur within the ultra micropores. Nevertheless the conversion of sulfur proceeds through polysulfides, however a clear distinction between long chain polysulfides at the beginning of discharge and short chain polysulfides at the end of discharge have been observed. The end discharge product is a mixture of $\mathrm{Li}_{2} \mathrm{~S}_{2}$ and $\mathrm{Li}_{2} \mathrm{~S}$ in the ratio 2:1. This is different compared to ether based electrolytes where a predominant end discharge phase is $\mathrm{Li}_{2} \mathrm{~S}$ in coexistence with mixture of different polysulfides.

\section{Acknowledgments}

Access to synchrotron radiation facilities of Elettra (project 20140487) is acknowledged. We would like to thank Luca Olivi of Elettra for expert advice on beamline operation. This research has received funding from the Slovenian Research Agency (research core funding No. P1-0112 and P2-0393), and from the European Union's Horizon 2020 research and innovation program under Grant Agreement No. 666221 (HELIS).

\section{ORCID}

Alen Vizintin (1D https://orcid.org/0000-0003-1876-1396

Lorenzo Stievano (D) https://orcid.org/0000-0001-8548-0231

\section{References}

1. B. Dunn, H. Kamath, and J.-M. Tarascon, Science, 334, 928 (2011).

2. P. G. Bruce, S. A. Freunberger, L. J. Hardwick, and J.-M. Tarascon, Nat. Mater, 11 19 (2011).

3. M. Hagen, D. Hanselmann, K. Ahlbrecht, R. Maça, D. Gerber, and J. Tübke, $A d v$ Energy Mater, 5, 1401986 (2015).
4. Y.-X. Yin, S. Xin, Y.-G. Guo, and L.-J. Wan, Angew. Chemie Int. Ed., 52, 13186 (2013).

5. Y. Yang, G. Zheng, and Y. Cui, Chem. Soc. Rev., 42, 3018 (2013).

6. A. Vizintin, M. U. M. Patel, B. Genorio, and R. Dominko, ChemElectroChem, 1 1040 (2014)

7. A. Vizintin, M. Lozinšek, R. K. Chellappan, D. Foix, A. Krajnc, G. Mali, G. Drazic B. Genorio, R. Dedryvère, and R. Dominko, Chem. Mater, 27, 7070 (2015).

8. X. Liang, Q. Pang, I. R. Kochetkov, M. S. Sempere, H. Huang, X. Sun, and L. F. Nazar, Nat. Energy, 2, 17119 (2017).

9. S. S. Zhang, Electrochim. Acta, 70, 344 (2012).

10. J. Scheers, S. Fantini, and P. Johansson, J. Power Sources, 255, 204 (2014).

11. M. Wild, L. O'Neill, T. Zhang, R. Purkayastha, G. Minton, M. Marinescu, and G. J. Offer, Energy Environ. Sci., 8, 3477 (2015).

12. R. Dominko, M. U. M. Patel, V. Lapornik, A. Vizintin, M. Koželj, N. N. Tušar, I. Arčon, L. Stievano, and G. Aquilanti, J. Phys. Chem. C, 119, 19001 (2015).

13. N. A. Cañas, S. Wolf, N. Wagner, and K. A. Friedrich, J. Power Sources, 226, 313 (2013).

14. S. Waluś, C. Barchasz, J.-F. Colin, J.-F. Martin, E. Elkaïm, J.-C. Leprêtre, and F. Alloin, Chem. Commun., 49, 7899 (2013)

15. M. A. Lowe, J. Gao, and H. D. Abruña, RSC Adv., 4, 18347 (2014).

16. J. Conder, R. Bouchet, S. Trabesinger, C. Marino, L. Gubler, and C. Villevieille, Nat. Energy, 2, 17069 (2017)

17. R. Xu, J. Lu, and K. Amine, Adv. Energy Mater, 5, 1500408 (2015).

18. M. Cuisinier, P.-E. Cabelguen, B. D. Adams, A. Garsuch, M. Balasubramanian, and L. F. Nazar, Energy Environ. Sci., 7, 2697 (2014).

19. M. U. M. Patel, I. Arčon, G. Aquilanti, L. Stievano, G. Mali, and R. Dominko, ChemPhysChem, 15, 894 (2014).

20. Y. Gorlin, M. U. M. Patel, A. Freiberg, Q. He, M. Piana, M. Tromp, and H. A. Gasteiger, J. Electrochem. Soc., 163, A930 (2016).

21. M. Cuisinier, C. Hart, M. Balasubramanian, A. Garsuch, and L. F. Nazar, Adv. Energy Mater, 5, 1401801 (2015).

22. A. Vizintin, L. Chabanne, E. Tchernychova, I. Arčon, L. Stievano, G. Aquilanti, M. Antonietti, T.-P. Fellinger, and R. Dominko, J. Power Sources, 344, 208 (2017).

23. T. A. Pascal, K. H. Wujcik, J. Velasco-Velez, C. Wu, A. A. Teran, M. Kapilashrami, J. Cabana, J. Guo, M. Salmeron, N. Balsara, and D. Prendergast, J. Phys. Chem. Lett., 5, 1547 (2014).

24. M. Kavčič, K. Bučar, M. Petric, M. Žitnik, I. Arčon, R. Dominko, and A. Vizintin, J. Phys. Chem. C, 120, 24568 (2016).

25. J. Hannauer, J. Scheers, J. Fullenwarth, B. Fraisse, L. Stievano, and P. Johansson, ChemPhysChem, 16, 2755 (2015)

26. N. Saqib, G. M. Ohlhausen, and J. M. Porter, J. Power Sources, 364, 266 (2017).

27. M. U. M. Patel, R. Demir-Cakan, M. Morcrette, J.-M. Tarascon, M. Gaberscek, and R. Dominko, ChemSusChem, 6, 1177 (2013).

28. M. U. M. Patel and R. Dominko, ChemSusChem, 7, 2167 (2014).

29. A. Di Cicco, G. Aquilanti, M. Minicucci, E. Principi, N. Novello, A. Cognigni, and L. Olivi, J. Phys. Conf. Ser, 190, 012043 (2009).

30. G. Aquilanti, M. Giorgetti, R. Dominko, L. Stievano, I. Arčon, N. Novello, and L. Olivi, J. Phys. D. Appl. Phys., 50, 074001 (2017).

31. B. Ravel and M. Newville, J. Synchrotron Radiat., 12, 537 (2005).

32. S. Drvarič, S. Jeschke, A. Vizintin, K. Pirnat, I. Arcon, G. Aquilanti, P. Johansson, and R. Dominko, Chem. Mater, 29(23), 10037 (2017).

33. C. Barchasz, J.-C. Leprêtre, S. Patoux, and F. Alloin, Electrochim. Acta, 89, 737 (2013).

34. S. Drvarič Talian, M. Bešter-Rogač, and R. Dominko, Electrochim. Acta, 252, 147 (2017).

35. L. Suo, Y.-S. Hu, H. Li, M. Armand, and L. Chen, Nat. Commun., 4, 1481 (2013).

36. L. Cheng, L. A. Curtiss, K. R. Zavadil, A. A. Gewirth, Y. Shao, and K. G. Gallagher, ACS Energy Lett., 1, 503 (2016).

37. S. Gu, R. Qian, J. Jin, Q. Wang, J. Guo, S. Zhang, S. Zhuo, and Z. Wen, Phys. Chem. Chem. Phys., 18, 29293 (2016)

38. N. Azimi, Z. Xue, I. Bloom, M. L. Gordin, D. Wang, T. Daniel, C. Takoudis, and Z. Zhang, ACS Appl. Mater. Interfaces, 7, 9169 (2015).

39. M. Helen, M. A. Reddy, T. Diemant, U. Golla-Schindler, R. J. Behm, U. Kaiser, and M. Fichtner, Sci. Rep., 5, 12146 (2015).

40. B. Zhang, X. Qin, G. R. Li, and X. P. Gao, Energy Environ. Sci., 3, 1531 (2010).

41. S. Zheng, P. Han, Z. Han, H. Zhang, Z. Tang, and J. Yang, Sci. Rep., 4, 4842 (2015)

42. T. Yim, M.-S. Park, J.-S. Yu, K. J. Kim, K. Y. Im, J.-H. Kim, G. Jeong, Y. N. Jo S.-G. Woo, K. S. Kang, I. Lee, and Y.-J. Kim, Electrochim. Acta, 107, 454 (2013). 